\title{
Double-outlet of Right Ventricle of Recipient Fetus in Severe Mid-trimester Twin-Twin Transfusion Syndrome
}

\author{
${ }^{1}$ Tachjaree Panchalee, ${ }^{2}$ Tuangsit Wataganara
}

\begin{abstract}
A 37 years old Thai pregnant woman with MCDA twins, para 1, was suspected of severe twin-twin transfusion syndrome (TTTS) at 21 weeks' of gestation. Quintero staging was applied in this cases. Neither of the fetuses exhibited signs of hydrops. Abnormal four-chamber view was demonstrated in a recipient fetus. Two-dimensional and color Doppler showed complex cardiac malformations which include (1) hypoplastic left ventricle, and (2) large atrial septal defect, (3) atretic tricuspid valve, and (4) the blind right atrium communicating with the left atrium through the ostiumprimum defect. For more information, threedimensional (3D) echocardiography was used toreconstruct"thin slab" of the fetal heart and its outflow tracts. A double-outletright ventricle (DORV) was highly suspicious. Congenital cardiac anomaly can decompensate the compromised myocardium of the recipient fetus. In addition to conventional 2D echocardiography, the application of advanced 3D echocardiography with 'thin slab' technique can help for the examination of fetal heart.
\end{abstract}

Keywords: Double outlet of right ventricle, Fetal echocardiography, Monochronic diamniotic twins, Twin-twin transfusion syndrome.

How to cite this article: Panchalee T, Wataganara T. Doubleoutlet of Right Ventricle of Recipient Fetus in Severe Midtrimester Twin-Twin Transfusion Syndrome. Donald School J Ultrasound Obstet Gynecol 2016;10(4):365-366.

Source of support: Nil

Conflict of interest: None

\section{INTRODUCTION}

A 37-year-old Thai pregnant woman, para 1, who conceived spontaneously, was transferred to our unit for a suspicion of severe twin-twin transfusion syndrome (TTTS) at 21 weeks' of gestation. Chorionicity was originally assigned as monochorionic diamniotic (MCDA) from her 1st trimester scan. Our ultrasound examination revealed oligopolyhydramniotic appearance. The donor fetus had a "stuck" appearance (very small urinary bladder and deepest liquor pool of $0.5 \mathrm{~cm}$ ). End-diastolic

\footnotetext{
${ }^{1}$ Lecturer, ${ }^{2}$ Associate Professor

1,2Division of Maternal-Fetal Medicine, Department of Obstetrics and Gynecology, Faculty of Medicine Siriraj Hospital, Bangkok Thailand

Corresponding Author: Tuangsit Wataganara, Associate Professor, Division of Maternal-Fetal Medicine, Department of Obstetrics and Gynecology, Faculty of Medicine Siriraj Hospital, Bangkok, Thailand, Phone: +011662 4197000, e-mail: twataganara@yahoo.com
}

flow in the umbilical artery of the donor fetus was positive. The recipient fetus had distended urinary bladder and increased liquor volume, with the deepest pool of $12 \mathrm{~cm}$. Neither of the fetuses exhibited signs of hydrops.

Recipient fetus had abnormal four-chamber view on the scan. Two-dimensional and color Doppler showed visceral situs solitus, levocardia, and complex cardiac malformations, which include (1) hypoplastic left ventricle, (2) large atrial septal defect, (3) atretic tricuspid valve, and (4) the blind right atrium communicating with the left atrium through the ostium primum defect. Pulmonary stenosis caused by the conal septal was seen. The ascending aorta was located anterior, to the right and parallel to the pulmonary trunk, which was smaller and also arose from the right ventricle (Figs 1A and B).

Three-dimensional (3D) echocardiography was used to reconstruct "thin slab" of the fetal heart and its outflow tracts (Fig. 2). Thin-slab technique is primarily used for image reconstruction from magnetic resonance imaging (MRI) of fetal cardiac structure (fetal cardiac MRI), but was informally used in this report as an analogy. Volume data sets were acquired using coronal sweeps through the fetal thorax. The acquisition was set to high quality, and the sweep angle was set to $35^{\circ}$. Volume data sets were initially displayed using multiplanar slicing, and the rendering mode was then applied to obtain the coronal reconstruction 3D echocardiogram. A combination of smooth-surface and gradient-light algorithms was adopted with surface
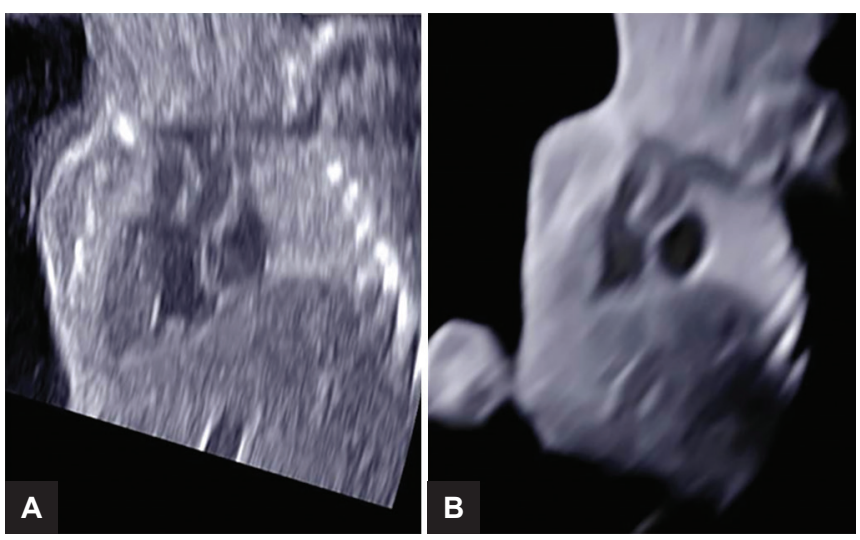

Figs 1A and B: Comparison of image quality for diagnosis of DORV in 21 weeks' recipient fetus of TTTS: (A) Conventional gray scale; and (B) 3D, surface-enhanced, "thin slab" technique for a better visualization of common origin of ascending aorta and main pulmonary artery from right ventricle 


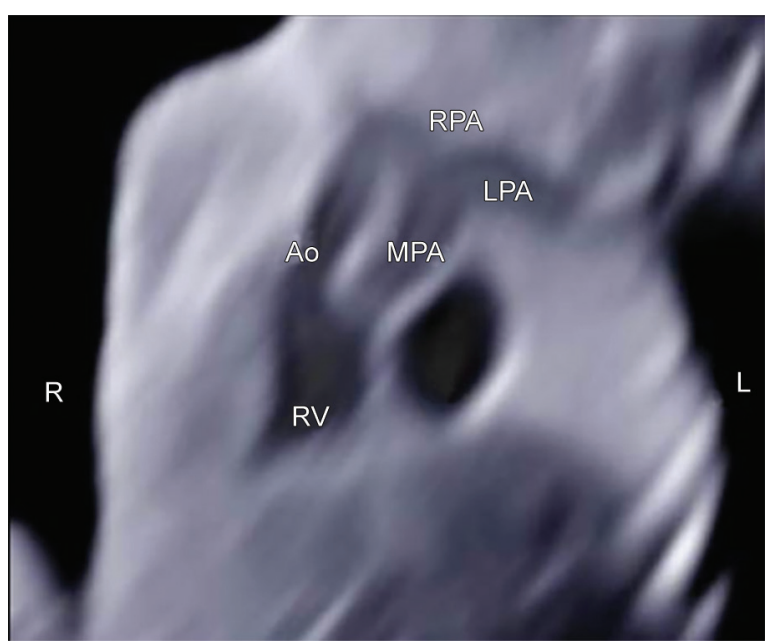

Fig. 2: Three-dimensional, surface-enhanced, "thin slab" technique demonstrated common origin of ascending aorta (Ao) and main pulmonary artery (MPA) from right ventricle (RV) (LA = left atrium, $\mathrm{RPA}=$ right main pulmonary artery, LPA = left main pulmonary artery, $L=$ left side of the fetus, $R=$ right side of the fetus)

rendering. The region of interest was adjusted, and rotation of the 4 D echocardiogram along the $\mathrm{x}-, \mathrm{y}-$, and $\mathrm{z}$-axes was performed until the structures of interest were visualized. Postprocessing adjustments were used to improve the image quality. The 4 D echocardiogram clearly showed the two great arteries originating in parallel from the right ventricle. A double-outlet right ventricle (DORV) was clearly shown (Fig. 2).

The patient was counselled for treatment options including (1) fetoscopic laser photocoagulation of chorionic anastomoses and (2) umbilical cord occlusion of the recipient fetus with DORV. Unfortunately, the patient broke her water soon after the examination. The patient and her family decided to abandon in utero intervention and to return to their primary hospital for further care. Both fetuses were aborted soon after that. Autopsy report of the recipient fetus showed two great arteries originate in parallel from the right ventricle, with the aorta on the right and the pulmonary artery on the left. The pulmonary trunk is smaller than aorta. Double-outlet right ventricle has been linked to chromosomal aneuploidies and 22q11 syndrome; but karyotype study and array comparative genomic hybridization $(\mathrm{aCGH})$ are not available in this case. ${ }^{2}$

In this report, we presented a rare case of DORV found in the recipient fetus of MCDA complicated with TTTS. Congenital cardiac anomaly can further jeopardize the readily compromised myocardium of the recipient fetus resulting from in utero volume overload. Our images depict the spatial relationship of the aorta and the pulmonary artery by 3D echocardiography. The essential part of the diagnosis of DORV depends on the exact analysis of the origin of the two great arteries, which was clearly shown in the 3D image reconstruction. Although DORV can be diagnosed by experienced sonographers with conventional 2D echocardiography, the application of advanced 3D echocardiography with surface-enhanced "thin slab" technique helps with the understanding and facilitate examination of the fetal heart.

\section{REFERENCES}

1. Yagel S, Cohen SM, Shapiro I, Valsky DV. 3D and 4D ultrasound in fetal cardiac scanning: a new look at the fetal heart. Ultrasound Obstet Gynecol 2007 Jan;29(1):81-95.

2. L'Herminé-Coulomb A, Houyel L, Aboura A, Audibert F, Dal Soglio D, Tachdjian G. Double-outlet right ventricle with absent left ventricle and mitral atresia in a fetus with a deletion 22q12. Prenat Diagn 2004;24:708-712. 